\title{
FREE NON-ASSOCIATIVE PRINCIPAL TRAIN ALGEBRAS
}

\author{
by P. HOLGATE
}

(Received 9th March 1984)

\section{Introduction}

The definitions of finite dimensional baric, train, and special train algebras, and of genetic algebras in the senses of Schafer and Gonshor (which coincide when the ground field is algebraically closed, and which I call special triangular) are given in WörzBusekros's monograph [8]. In [6] I introduced applications requiring infinite dimensional generalisations. The elements of these algebras were infinite linear forms $\sum x_{i} a_{i}$ in basis elements $a_{0}, a_{1}, \ldots$ and complex coefficients such that $\sum_{i=0}^{\infty}\left|x_{i}\right|<\infty$. In this paper I consider only algebras whose elements are forms $\sum x_{i} a_{i}$ for which only a finite number of the $x_{i}$ are non zero. An algebra $\mathfrak{X}$, finite or infinite dimensional, not necessarily associative, but for the purpose of this article commutative, is said to be baric if it admits a homomorphism $w$ into its ground field. For $g \in \mathfrak{U}, w(g)$ is called the weight of $g$. The kernel of $w$, which has codimension 1 in $\mathfrak{U}$ is denoted by $\mathfrak{R}$. The principal powers of $g$ are defined by $g^{1}=g, g^{j+1}=g^{j} g$. If, for all $g$ with $w(g)=1$, they satisfy a rank equation

$$
r(g)=\sum_{j=1}^{n+1} \theta_{j} g^{j}=0
$$

for some fixed set of constants $\theta_{1}, \ldots, \theta_{n+1}, \mathfrak{U}$ will be called $a$ strong principal train algebra. The roots of the auxiliary scalar equation $\sum_{j=1}^{n+1} \theta_{j} x^{j-1}=0$ are called the principal train roots of $\mathfrak{A}$. It follows from the properties of the homomorphism that for all $g \in \mathfrak{A}, \sum \theta_{j} w^{n+1-j}(g) g^{j}=0$. If for an infinite dimensional $\mathfrak{A}$, every finite dimensional homomorphic image is a strong principal train algebra, $\mathfrak{U}$ itself will be called a weak principal train algebra. If there is a strictly decreasing sequence of ideals $\left\{\boldsymbol{\Omega}_{\boldsymbol{j}}\right\}, j=0,1,2, \ldots$ in $\mathfrak{U}$ such that

$$
\mathfrak{R}_{o}=\mathfrak{A}, \mathfrak{R}_{1}=\mathfrak{\Re}, \boldsymbol{\Omega}_{j+1} \subset \mathfrak{R}_{j}, \mathfrak{R}_{1} \boldsymbol{R}_{j} \subseteq \mathfrak{R}_{j+1}, \quad j=1,2,3, \ldots
$$

then $\mathfrak{U}$ is said to be weakly special triangular. When such a sequence exists it is possible to find a sequence of ideals satisfying (2), and such that for all $j, \Omega_{j+1}$ has codimension 1 in $\boldsymbol{R}_{j}$. Thus $\mathfrak{U}$ admits a canonical basis $c_{0}, c_{1}, \ldots$ such that $c_{0}, c_{j} \in \mathfrak{R}_{j}$ while for $1 \leqq i \leqq j$, $c_{i} c_{j} \in \Omega_{j+1}$. The numbers $\lambda_{o j j}$ such that $c_{0} c_{j}-\lambda_{o j j} c_{j} \in \Omega_{j+1}$ are called the train roots of $\mathfrak{U}$. They are the common eigenvalues of multiplications by $a \in \mathfrak{U}, w(a)=1)$. Note that the ordering of the canonical basis implies a corresponding ordering of the train roots. If for every sequence of ideals satisfying (2), we have $\bigcap_{j=0}^{\infty} \mathfrak{R}_{j}^{\prime}=0, \mathfrak{U}$ is said to be strongly 
special triangular. Finally, if the principal powers $\boldsymbol{\Omega}^{j}$ of $\boldsymbol{\Omega}$ form a sequence of ideals with the above property, $\mathfrak{A}$ is said to be a weak or strong special train algebra respectively.

For finite dimensions, the special train property implies special triangularity which implies the principal train property. The train roots of special triangular algebras occur as their principal train roots, possibly with reduced multiplicities, except that $\frac{1}{2}$ may be a train root without arising as a principal train root [8, Theorems 3.10, 3.29]. Special triangular algebras that are not special train are easy to construct, and arise in applications [8, Theorem 3.30], but the existence of commutative train algebras over fields of characteristic not 2 , which are not special triangular, was only settled by Abraham's examples [1].

If, in a baric algebra $\mathfrak{U}$, the plenary powers of every element $g \in \mathfrak{U}$ with $w(g)=1$, defined by $g^{[1]}=g, g^{[j+1]}=\left(g^{[j]}\right)^{2}$, satisfy a linear recurrence relation

$$
\sum_{j=1}^{t} \beta_{j} g^{[j]}=0, \text { for fixed } \beta_{1}, \ldots, \beta_{t}
$$

then $\mathfrak{U}$ is said to satisfy the plenary train condition. It follows that for all $g \in \mathfrak{A}$, $\sum_{j=1}^{t} \beta_{j} w^{2^{t-j}}(g) g^{[j]}=0$.

The relations between the classes of algebras defined above, and the conditions under which the plenary train, and similar conditions are satisfied, have been studied in a number of papers in the finite dimensional case, for which the following results hold (for references see [8]).

In [3] I showed that all special triangular algebras that contain idempotents satisfy the plenary train condition, although the theorem was stated for special train algebras. The requirement of an idempotent is however superfluous and can easily be removed from the proof. Recently, McHale and Ringwood [7] have shown that an equation (3) can be constructed by a particular technique which they call Haldane linearisation, if and only if $\mathfrak{U}^{[s]}$ is special triangular for some integer $s$.

\section{Free non-associative algebras}

The commutative, non-associative powers of $a$, of degree $i$, will be denoted by $\left\{a_{i j}\right\}$, $i=1,2, \ldots ; j=1,2, \ldots b_{i}$ where $i$ is the degree of the power, and $b_{i}$ the number of such powers, of degree $i$. They may be ordered, beginning with $a_{11}=a, a_{21}=a^{2}, a_{31}=a^{3}$, $a_{41}=a^{4}, a_{42}=\left(a^{2}\right)^{2}, \ldots$ In general, $a_{i j}$ precedes $a_{r s}$ if $i<r$. If $i=r$, consider the unique decompositions of $a_{i j}, a_{i s}$ into pairs of factors of lower degree, already placed in sequence. Then $a_{i j}$ precedes $a_{i s}$ if it gives rise to the earliest of the four factors, or if each power gives rise to one of a pair of jointly earliest factors, and the remaining factor of the $a_{i j}$ precedes that of $a_{i s}$. (A similar procedure is followed to order the noncommutative, non-associative powers, except that ordering within a degree is by the first factor, then by the second). Note that $a_{i 1}=a^{i}$, the ith principal power. The free commutative, non-associative algebra without identity, with one generator, consists of finite forms $\sum x_{i j} a_{i j}$, with coefficients in some field, which will be taken to be the complex numbers, with the natural multiplication. It will be denoted by $\mathfrak{F}_{1}$. The corresponding algebra $\mathfrak{F}_{k}$, with $k$ generators $a, b, c \ldots$ consists of finite linear forms in non-associative products of these symbols. 
Theorem 1. The algebra $\mathfrak{F}_{k}$ is baric for every $k$. It is strongly special triangular when $k=1$, but not weakly special train. For $k \geqq 2$ it is weakly but not strongly special triangular.

Proof. For $k=1, w(a)=\theta$ generates a weight function. If $\theta \neq 1$ we take $a^{*}=\theta^{-1} a$ as generator, so the weight function is essentially unique. For $k \geqq 2$ there are $2^{k}-1$ different weight functions obtained by assigning weight 1 to subsets of the generating symbols.

For $k=1$, we construct a sequence of ideals satisfying the requirements of special triangularity. Write

$$
\begin{aligned}
& c_{11}=a_{11} \\
& c_{i 1}=a_{i 1}-a_{i-1,1}=a^{i}-a^{i-1}, \text { for } i>1 \\
& c_{i j}=a_{i j}-a_{i 1}=a_{i j}-a^{i}, \text { for } i>1, j>1,
\end{aligned}
$$

and order the $c_{i j}$ as the corresponding $a_{i j}$. Then

(i) $c_{11} c_{i 1}=a\left(a^{i}-a^{i-1}\right)=a^{i+1}-a^{i}=c_{i+1,1}$

(ii) $c_{11} c_{i j}=a\left(a_{i j}-a^{i}\right)=a a_{i j}-a^{i+1}=c_{i+1, s}$, when $j>1$, for some $s>1$, since $a a_{i j}$ is not a principal power.

(iii) $c_{i 1} c_{j 1}=\left(a^{i}-a^{i-1}\right)\left(a^{j}-a^{j-1}\right)$

$$
\begin{aligned}
= & \left(a^{i} a^{j}-a^{i+j}\right)-\left(a^{i} a^{j-1}-a^{i+j-1}\right)-\left(a^{i-1} a^{j}-a^{i+j-1}\right) \\
& +\left(a^{i-1} a^{j-1}-a^{i+j-2}\right)+\left(a^{i+j}-a^{i+j-1}\right)-\left(a^{i+j-1}-a^{i+j-2}\right) \\
= & c_{i+j, u}-c_{i+j-1, v}-c_{i+j-1, w}+c_{i+j-2, p}+c_{i+j, 1}-c_{i+j-1,1}
\end{aligned}
$$

where $i, j>1$, for some $u, v, w, p>1$.

(iv) $c_{i 1} c_{j k}=\left(a^{i}-a^{i-1}\right)\left(a_{j k}-a^{j}\right)$

$$
\begin{aligned}
= & \left(a^{i} a_{j k}-a^{i+j}\right)-\left(a^{i} a^{j}-a^{i+j}\right)-\left(a^{i-1} a_{j k}-a^{i+j-1}\right) \\
& +\left(a^{i-1} a^{j}-a^{i+j-1}\right) \\
= & c_{i+j, u}-c_{i+j, v}-c_{i+j-1, w}+c_{i+j-1, p}
\end{aligned}
$$

where $i, j, k>1$, for some $u, v, w, p>1$.

(v) $c_{i l} c_{j k}=\left(a_{i l}-a^{i}\right)\left(a_{j k}-a^{j}\right)$

$$
\begin{aligned}
& =\left(a_{i l} a_{j k}-a^{i+j}\right)-\left(a_{i l} a^{j}-a^{i+j}\right)-\left(a^{i} a_{j k}-a^{i+j}\right)+\left(a^{i} a^{j}-a^{i+j}\right) \\
& =c_{i+j, u}-c_{i+j, v}-c_{i+j, w}+c_{i+j, p}
\end{aligned}
$$

where $i, j, k, l>1$, for some $u, v, w, p>1$. 
Now take $\Omega_{i j}$ to be the subspace spanned by $c_{i j}$ and succeeding $c_{s t}$ 's. (In this notation $\mathfrak{F}_{1}=\mathfrak{R}_{11}, \Omega_{21}$ is the kernel of the weight function.) The above calculations show that with the assigned ordering of the $c_{i j}$, the $\boldsymbol{R}_{i j}$ form' a sequence of ideals satisfying (2). Since every element is a finite linear combination of the $a_{i j}, \cap \boldsymbol{R}_{i j}=0$. Moreover, $w$ and hence its kernel are unique. Any sequence $\left\{\boldsymbol{\Re}_{t}^{\prime}\right\}$ of ideals satisfying (2) must satisfy $\boldsymbol{\Omega}_{t}^{\prime} \subseteq\left(t\right.$ th member of $\left.\left\{\boldsymbol{\Omega}_{i j}\right\}\right)$, and hence $\cap \boldsymbol{R}_{t}^{\prime}=0$.

To obtain a basis for $\mathfrak{F}_{k}$, each $a_{i j}$ is replaced by $k^{i}$ terms formed by substituting for each factor " $a$ ", each of the $k$ generators. To order them we return to the noncommutative, non-associative powers. These are first ordered as described above, and then the different products corresponding to each power are ordered lexicographically, and any term equal to a preceding term by commutativity is deleted. These terms are denoted by $t_{i j l}$. We form $c_{i 11}=t_{i 11}-a^{i-1}=a_{i}-a^{i-1} ; c_{i j 1}=t_{i j 1}-t_{1 j 1}=a_{i j}-a^{i}, j \neq 1 ; c_{i j l}=$ $t_{i j l}, l \neq 1$. Denote by $\boldsymbol{\Omega}_{i j l}$ the subspace spanned by $c_{i j l}$ and succeeding elements. Then $\left\{\mathfrak{\Omega}_{i j l}\right\}$ satisfies (2). However although $\cap \boldsymbol{\Re}_{i j l}=0$, consider the ideals $\mathfrak{L}_{\infty}$ spanned by $t_{i j l}$, $l \neq 1$, i.e. all basis elements containing a factor other than $a$, and $\mathfrak{D}_{i j}$ spanned by these together with all terms in powers of $a$ alone from $c_{i j}$ onwards. Then $\left\{\boldsymbol{I}_{i j}\right\}$ is a sequence of ideals satisfying (2), with $\cap \mathfrak{R}_{i j}=\mathfrak{L}_{\infty} \neq 0$.

The algebra $\mathfrak{F}_{1}$ fails at the first test for the special train property, since $\Omega_{21}^{2}$ is not an ideal. To prove this consider the quotient algebra $\mathfrak{F}_{1} / \mathfrak{R}_{61}$. If the cosets of $\boldsymbol{R}_{61}$ are represented by elements $c_{11}, c_{21}, c_{31}, c_{41}, c_{42}, c_{51}, c_{52}, c_{53}$, the multiplication table is found by direct computation to be

\begin{tabular}{l|llllc}
\multicolumn{1}{c}{$c_{11}$} & $c_{21}$ & $c_{31}$ & $c_{41}$ & $c_{42}$ \\
\cline { 2 - 6 }$c_{11}$ & $c_{11}+c_{21}$ & $c_{31}$ & $c_{41}$ & $c_{51}$ & $c_{52}$ \\
$c_{21}$ & & $c_{41}+c_{42}-c_{31}$ & $-c_{41}-c_{42}+c_{51}+c_{53}$ & $-c_{51}-c_{52}$ & $-c_{52}$ \\
$c_{31}$ & & & $c_{42}-c_{51}-2 c_{53}$ & 0 & 0
\end{tabular}

and all other products zero. This is baric with $\Omega_{21} / \Omega_{61}$ the kernel of the weight function. Its square $\left(\boldsymbol{\Omega}_{21} / \boldsymbol{\Omega}_{61}\right)^{2}$ is spanned by $c_{31}-c_{41}-c_{42}, c_{41}+c_{42}-c_{53}, c_{42}-2 c_{53}, c_{51}, c_{52}$. Now $c_{11}\left(c_{21}^{2}\right)=c_{11}\left(c_{41}-c_{42}-c_{31}\right)=c_{51}-c_{52}-c_{42} \notin\left(\boldsymbol{\Omega}_{21} / \boldsymbol{\Omega}_{61}\right)^{2}$. Thus $\left(\boldsymbol{\Omega}_{21} / \boldsymbol{\Omega}_{61}\right)^{2}$ is not an ideal in $\Omega_{11} / \Re_{61}$, and hence $\Re_{21}^{2}$ cannot be an ideal in $\boldsymbol{R}_{11}$.

\section{Free train algebras}

Now let $\Re_{k, n}=\Re_{k, n}\left(\theta_{1}, \ldots, \theta_{n+1}\right)$ be the ideal in $\mathfrak{F}_{k}$ generated by elements $r(g)=$ $\sum_{j=1}^{n+1} \theta_{j} w^{n+1-j}(g) g^{j}, g \in F_{k}$. The quotient $\mathfrak{F}_{k} / \mathfrak{R}_{k, n}$, denoted by $\mathfrak{F}_{k, n}\left(\theta_{1}, \ldots, \theta_{n+1}\right)$ will be called the free commutative non-associative principal train algebra without identity, corresponding to the principal train equation (1). By routine calculation, its elements can be seen to satisfy (1), while every commutative, non-associative principal train algebra without identity, with principle train equation (1), is a homomorphic image of $\mathfrak{F}_{k, n}$.

Lemma. The strong special triangular property is preserved under homomorphism.

Proof. Let $\mathfrak{R}_{o} \supset \boldsymbol{R}_{1} \supset \mathfrak{R}_{2} \supset \ldots$ be a sequence of ideals in $\mathfrak{A}$ satisfying (2) and $\cap \boldsymbol{R}_{i}=\phi$, and let $\mathfrak{R}$ be the kernel of the homomorphism from $\mathfrak{A}$ to $\mathfrak{U}^{*}$. If $\mathfrak{\Omega}_{i}^{*}$ is the image of $\boldsymbol{\Omega}_{i}$, 


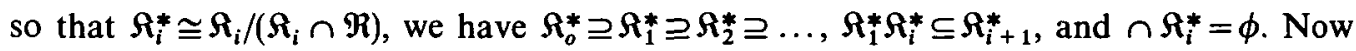
delete all but the first of any segments of equal members of $\left\{\Omega_{i}^{*}\right\}$. The remaining sequence shows that $\mathfrak{U}^{*}$ is strongly special triangular.

Corollary to Theorem 1 . Let $\mathfrak{R}$ be any ideal of $\mathfrak{F}_{1}$. Then $\mathfrak{F}_{1} / \mathfrak{R}$ is strongly special triangular. Hence any algebra with a single generator is special triangular.

Theorem 2. For $k=1, n=1,2, \mathfrak{F}_{k, n}$ is finite dimensional. For $k=1, n \geqq 3, \mathfrak{F}_{k, n}$ may be infinite dimensional.

Proof. For $n=1$ the principal train equation must be $g-g^{2}=0$. In particular $a^{2}=a$ and hence all $a_{i j}=a\left(\bmod \mathfrak{R}_{1,1}\right)$. Thus $\mathfrak{F}_{1} / \mathfrak{R}_{1,1}$ is homomorphic to the ground field.

For $n=2$ consider the principal train equation $g^{3}-(1-\lambda) g^{2}-\lambda g=0$. Let $b, c, d$ be three of the $a_{i j}$, not necessarily distinct. If we set $g=(\theta b+\phi c+\psi d) /(\theta+\phi+\psi)$ and after multiplying by $(\theta+\phi+\psi)^{3}$, equate to zero the term in $\theta \phi \psi$, we have $\{(b c) d+(d b) c+$ (cd) $b\}-(1-\lambda)(c d+b c+d b)-\lambda(b+c+d)=0$. On substituting $(b, c, d)=\left(a, a, a^{2}\right)$ and working $\bmod \Re_{2}$, we obtain $\left(a^{2}\right)^{2}+2 a^{4}-2(1-\lambda) a^{3}-a^{2}-2 \lambda a=0$. Now $a^{3}=\lambda a+(1-\lambda) a^{2}$ which implies $a^{4}=\lambda a^{2}+(1-\lambda) a^{3}$, and the above relation leads to $\left(a^{2}\right)^{2}=2 \lambda a+(1-2 \lambda) a^{2}$. Thus all 4th powers are congruent to forms of strictly lower degree, and in fact of degree 2. The multiplication table of $\mathfrak{F}_{1} / \mathfrak{R}_{1,2}$ in this case is $\bmod \mathfrak{R}_{1,2}$.

$$
\begin{array}{lll}
\multicolumn{1}{l}{} & \multicolumn{1}{l}{a} & a^{2} \\
\cline { 2 - 3 } a & a^{2} & \lambda a+(1-\lambda) a^{2} \\
a^{2} & & 2 \lambda a+(1-2 \lambda) a^{2}
\end{array} .
$$

By straightforward calculation we find that the plenary powers of $a$ are given by

$$
a^{[s]}=\left\{2 \lambda a+a^{2}+(-2 \lambda)^{s-1}\left(a-a^{2}\right)\right\} /(1+2 \lambda) .
$$

Finally, consider the principal train equation $g-g^{4}=0, w(g)=1$, and the corresponding algebra $\mathfrak{F}_{1} / \mathfrak{R}_{1,3}$. We show that its dimension $d$ is infinite. First, $d$ must exceed 3 . The elements $a, a^{2}, a^{3}$ are linearly independent, and if $d=3$ we would have $\left(a^{2}\right)^{2}=(1-\theta-\phi) a+$ $\theta a^{2}+\phi a^{2}\left(\bmod \mathfrak{R}_{1,3}\right)$ for some $\theta, \phi$. That is,

$$
\left(\left(a^{2}\right)^{2}-a\right)-\theta\left(a^{2}-a\right)-\phi\left(a^{3}-a\right) \in \mathfrak{R}_{1,3} .
$$

But $\Re_{1,3}$ consists of finite sums of terms, each of which contains a factor $\left(g_{i}^{4}-g_{i}\right)$. For such expressions, if the leading term is of degree 4 , it must be a principal product of 4 factors, and hence the element displayed above does not belong to $\Re_{1,3}$. The principal train roots of $\mathfrak{F}_{1} / \Re_{1,3}$ are $1, \omega, \omega^{2}$ and are simple. If $3<d<\infty$ there must be train roots (that is, common eigenvalues of the operation of multiplication by $g, w(g)=1$ ) other than these. Each of these additional train roots involves an eigenrelationship that is not implied by $\mathfrak{R}_{1,3}$, and hence the algebra is not a free principal train algebra. Thus $d$ cannot exceed 3 . The contradiction shows that $d$ is infinite. 


\section{The plenary train condition}

Theorem 3. Every finite dimensional principal train algebra satisfies the plenary train condition.

Proof. Let $\mathfrak{U}$ be a principal train algebra of finite dimension $n$, and principal train roots $\lambda_{1}, \ldots, \lambda_{s}$. Denote by $\mathfrak{U}_{g}$ the algebra generated by $g \in \mathfrak{A}, w(g)=1$. By the Corollary to Theorem 1 it is special triangular, and as a subalgebra its principal train roots must lie among $\lambda_{1}, \ldots, \lambda_{s}$. Hence its train roots must have values among $\frac{1}{2}, \lambda_{1}, \ldots, \lambda_{s}$. A special triangular algebra with an ordered set of train roots $\mu_{1}, \mu_{2}, \ldots, \mu_{n}$ (repetitions included separately) satisfies the plenary train condition (see end of Section 1), with plenary train roots lying among the values $\mu_{1}^{r_{1}} \ldots \mu_{n}^{r_{n}}$, where $\sum 2^{i-1} r_{i} \leqq 2^{n-1}$ (see [2], Proposition 1, p. 356 , where the plenary roots belong to the monomials listed). Since we know neither the multiplicities nor the ordering of the train roots of $\mathfrak{A}_{g}$, we can only assert that its plenary train roots lie among the set of values $\left(\frac{1}{2}\right)^{r_{o}}, \lambda_{1}^{r_{1}}, \ldots, \lambda_{s}^{r_{s}}$, with $r_{i} \leqq n 2^{n-1}$. Denote this set of values by $v_{1}, \ldots, v_{m}$, with repetitions. The plenary train equation of $\mathfrak{A}_{g}$ is a factor of $\left\{\prod_{i=1}^{m}\left(E-v_{i}\right)\right\} g=0, w(g)=1$, where $E g=g^{2}$. Similarly, every $g \in \mathfrak{A}, w(g)=1$ satisfies this equation. Thus $\mathscr{U}$ satisfies the plenary train condition and its plenary train equation is also a factor of it.

Consider the second example given by Abraham [1] of a principal train algebra that is not special triangular. It has basis $c_{0}, c_{1}, \ldots, c_{5}$ with multiplication table

\begin{tabular}{l|cccccc}
\multicolumn{1}{c}{} & $c_{0}$ & $c_{1}$ & $c_{2}$ & $c_{3}$ & $c_{4}$ & $c_{5}$ \\
\cline { 2 - 7 }$c_{0}$ & $c_{0}$ & $\frac{1}{2} c_{1}$ & $\frac{1}{2} c_{2}$ & $\frac{1}{2} c_{3}$ & $\frac{1}{2} c_{4}$ & $\frac{1}{2} c_{5}$ \\
$c_{1}$ & & 0 & $c_{3}$ & $c_{4}$ & 0 & $-c_{3}$ \\
$c_{2}$ & & & 0 & $c_{5}$ & $c_{3}$ & 0
\end{tabular}

and all other products zero. On squaring a general element of weight 1 we find

$$
\begin{aligned}
\left(c_{0}+\sum_{1}^{5} x_{i} c_{i}\right)^{2}= & c_{o}+\sum_{1}^{5} x_{i} c_{i}+\left(2 x_{1} x_{2}-2 x_{1} x_{5}+2 x_{2} x_{4}\right) c_{3} \\
& +2 x_{1} x_{3} c_{4}+2 x_{2} x_{3} c_{5} .
\end{aligned}
$$

The sub-algebra generated by this element intersects the sub-space spanned by $c_{o}, c_{1}, c_{2}$ in multiples of $c_{o}+x_{1} c_{1}+x_{2} c_{2}$. Thus the algebra has no single generator and in fact requires three. The algebra satisfies $\mathfrak{Q}^{2}=\mathfrak{U}$ and thus by McHale and Ringwood's result [8], the construction of a plenary train equation by Haldane linearisation of the operator $E$, which generates the plenary powers by $g E=g^{2}$, is impossible. Let us nevertheless attempt to linearise $E$, by the methods introduced in [3], studied further in [2] and exploited in $[4,5]$. The above equation exhibits the effect of $E$ on coordinates:

$$
\begin{gathered}
x_{1} E=x_{1}, x_{2} E=x_{2}, x_{3} E=x_{3}+2\left(x_{1} x_{2}-x_{1} x_{5}+x_{2} x_{4}\right) \\
x_{4} E=x_{4}+2 x_{1} x_{3}, x_{5} E=x_{5}+2 x_{2} x_{3} .
\end{gathered}
$$


We adjoin further "coordinates" set equal to $x_{1} x_{3}, x_{2} x_{3}, x_{1} x_{2}-x_{1} x_{3}+x_{2} x_{4}(=u$, say). The effect of $E$ on these is

$$
\left(x_{1} x_{3}\right) E=x_{1} x_{3}+2 x_{1} u,\left(x_{2} x_{3}\right) E=x_{2} x_{3}+2 x_{2} u, \quad u E=u,
$$

the last relation appearing after some cancellation. At the next stage we add in further "coordinates" $x_{1} u, x_{2} u$ for which $\left(x_{1} u\right) E=x_{1} u,\left(x_{2} u\right) E=x_{2} u$. In the terminology of [7] we project points $\left(1, x_{1}, \ldots, x_{5}\right)$ of $\mathfrak{U}$ into $\left(1, x_{1}, \ldots, x_{5}, x_{1} x_{3}, x_{2} x_{3}, u, x_{1} u, x_{2} u\right)$ in a space $\hat{\mathfrak{U}}$. The operator $E$ on $\mathfrak{A}$ is now represented by a linear operator $\hat{E}$ on $\hat{\mathfrak{A}}$. In this case $\hat{E}$ has 1's on its main diagonal, and 2's in positions corresponding to $\left(x_{3}, u\right),\left(x_{4}, x_{1} x_{3}\right)$, $\left(x_{5}, x_{2} x_{3}\right),\left(x_{1} x_{3}, x_{1} u\right)$ and $\left(x_{2} x_{3}, x_{2} u\right)$. It has minimal polynomial $(\hat{E}-1)^{3}=0$, which is equivalent to the plenary train equation $g^{[4]}-3 g^{[3]}+3 g^{[2]}-g=0$. It would seem that if the process of Haldane linearisation studied by McHale and Ringwood had been carried through, we would have had to include at the first stage, coordinates equal to $x_{1} x_{2}$ and $x_{2} x_{4}$. Since $\left(x_{2} x_{4}\right) E=x_{2} x_{4}+2 x_{1} x_{2} x_{3}$, a coordinate $x_{1} x_{2} x_{3}$ would be needed at the next stage, and this would lead to an infinite sequence of additional coordinates.

In response to the question left open by $\mathrm{McH}$ Hale and Ringwood [7] in their last sentence, Theorem 3 and Abraham's example show that there is a class of algebras where $E$ can be linearised, by following informally the methods used by Haldane, but not by the precisely defined technique of "Haldane linearisation" with which they work, and for which they establish necessary and sufficient conditions.

This work has benefited greatly from discussions with David McHale and Graem Ringwood, and in particular from Graem Ringwood's critical reading of an earlier draft. Their respective studentship and Research Assistantship were financed by the SERC.

\section{REFERENCES}

1. V. M. Abraham, A note on train algebras, Proc. Edinburgh Math. Soc. (2) 20 (1976), 53-58.

2. V. M. Abraham, Linearising quadratic transformations in genetic algebras, Proc. London Math. Soc. (3) 40 (1980), 346-363. The induced linear transformation in a genetic algebra, ibid. 364-384.

3. P. Holgate, Sequences of powers in genetic algebras, J. London Math. Soc. 42 (1967), 489-496.

4. P. Holgate, Selfing in genetic algebras, J. Math. Biol. 6 (1978), 197-206.

5. P. Holgate, Genetic algebras for some inbreeding systems, Proc. Royal Soc. Edinburgh A 86 (1980), 65-74.

6. P. Holgate, Population algebras, J. Royal Statist. Soc. B 43 (1981), 1-19.

7. D. MCHale and G. A. Ringwood, Haldane linearisation of baric algebras, J. London Math. Soc. (2) 28 (1983), 17-26.

8. A. Worz-Busekros, Algebras in genetics (Lectures Notes in Biomathematics 36, Springer Verlag, 1980).

Birkbeck College

LONDON WCIE 7HX 